\title{
Impact of the plasma power on plasma-induced increase in absorption of fused silica
}

\author{
Christoph Gerhard*, Emilie Letien, Thomas Cressent and Mandy Hofmann
}

\section{Zusammenfassung}

Die Laserbearbeitung von Quarzglas hat in den letzten Jahrzehnten an Bedeutung gewonnen. Dabei besteht die Herausforderung darin, die hohe Transmission dieses Mediums zu überwinden. Hierzu stellt eine Plasmabehandlung mittels wasserstoffhaltigen Prozessgasen einen Ansatz dar. Dabei wird eine oberflächennahe Glasschicht durch Entfernung von Sauerstoff und Implantation von Wasserstoff chemisch modifiziert, woraus die Ausbildung optisch aktiver Defekte und ein Anstieg der Absorption resultieren. Vor diesem Hintergrund wurde in dieser Arbeit der Einfluss der Plasmaleistung auf die plasmainduzierte Absorptionssteigerung untersucht. Die höchste Effizienz der Plasmabehandlung wurde bei der geringsten applizierten Leistung erzielt, wohingegen bei höheren Plasmaleistungen nennenswert geringere $A b-$ sorptionssteigerungen beobachtet wurden. Dieser Effekt wird auf den Zerfall von Plasmaspezies, die für eine Ausbildung optisch aktiver Defekte benötigt werden, zurückgeführt, welcher mit ansteigender Leistung und dem damit einhergehenden Anstieg der elektrischen Feldstärke im Plasma anwächst. Diese Erkenntnis ist von hohem Interesse für ein umfassendes Verständnis von Plasma-Glas-Wechselwirkungen.

\section{Abstract}

In the last decades, laser machining of fused silica has gained in importance where the main challenge is to overcome the high transmission of this medium in order to achieve surface absorption. One approach is plasma treatment using hydrogenous process gases. In doing so, a nearsurface glass layer is chemically modified by a removal of oxygen and an implantation of hydrogen, resulting in the formation of optically active defects and an increase in absorption. Against this background, the impact of the plasma power on plasma-induced increase in absorption of fused silica was investigated in the present work. Here, the highest efficiency of the plasma treatment process was found at the lowest applied plasma power whereas for higher plasma powers, a notably lower increase in absorption was observed. This effect is attributed to the decomposition of plasma species required for initiating the formation of optically active defects within the glass. Such decomposition increases with rising power due to the corresponding increase in electric field strength within the plasma. This finding is of substantial interest for an extensive understanding of plasma-glass interactions.

\section{Résumé}

Ces dernières décennies, les applications laser en usine composées de silice fondue ont gagné en importance, I'enjeu consistant à surmonter le problème de la transmission élevée de ce milieu dans le but d'obtenir l'absorption de surface. Une façon d'aborder le problème est d'appliquer le traitement au plasma en utilisant des procédés au gaz de dihydrogène. En agissant ainsi, une couche de verre proche de la surface est modifiée chimiquement par l'ablation d'oxygène et I'implantation d'hydrogène, ce qui provoque la formation de défauts optiquement actifs et l'augmentation de l'absorption. Dans ce contexte, l'impact de la puissance du plasma sur l'augmentation de l'absorption de silice fondue induite par le plasma a été étudié dans cet article. Ici, la meilleure efficacité du traitement au plasma a été constatée à la puissance de plasma la plus faible tandis que pour de plus fortes puissances, une augmentation bien plus faible en absorption a été observée. Cet effet est attribué à la décomposition des espèces de plasma requises dans la formation des défauts optiquement actifs à l'intérieur du verre. Une telle décomposition augmente avec la montée de puissance due à l'augmentation de force du champ électrique dans le plasma. Cette découverte est d'un intérêt considérable pour une compréhension plus poussée des interactions plasma-verre. 


\section{Introduction}

Due to its unique properties, fused silica is one of the most important glasses in modern optical technology. From a manufacture-related point of view, laser-based free form structuring of this medium is of great interest for a number of different fields of application in optics and photonics as for example for the generation of micro lens arrays or diffractive micro optical elements. In the context of laser materials processing, the main advantage of fused silica - its high transparency in the ultraviolet (UV) wavelength range - becomes a challenging task; the dominating bulk absorption gives rise to poor machinability of fused silica surfaces. Quite different approaches were developed in the last few decades in order to overcome this issue and to achieve sufficient near-surface absorption for coupling the energy of incident laser light into the material lattice. Apart from the use of ultrashort laser pulses with pulse durations in the pico- or femtosecond range (Utéza et al. 2011), combined techniques based on the application of absorbing coatings on fused silica surfaces are in hand. Here, laser etching at a surface adsorbed layer (LESAL) (Böhme \& Zimmer 2004), laser-induced backside wet etching (LIBWE) (Wang et al. 1999), and laser-induced backside dry etching (LIBDE) (Hopp et al. 2006) shall be mentioned.

The latter technique is normally based on the deposition of solid absorbing layers, e.g. made of metal or silicon monoxide. However, increasing surface absorption can also be achieved by another novel approach which was presented in previous work (Brückner et al. 2012, Hoffmeister et al. 2012). Here, a near-surface layer of the glass network is modified by atmospheric pressure plasma treatment due to a chemical reduction of the glass-forming silicon dioxide and the additional implantation of hydrogen (Gerhard et al. 2012, Tasche et al. 2014) ${ }^{1}$. The resulting optically active defects within the glass such as oxygen deficiency centres, E'-centres or hydrogen centres feature high absorption in the

ultraviolet wavelength range, mainly from approximately 170 to $350 \mathrm{~nm}$ (Skuja 1998). In order to initiate such defects and an accompanying increase in absorption, the application of lowtemperature plasmas and the use of hydrogenous process gases has turned out to be a suitable and powerful method (Gerhard et al. 2013).

The performance of such plasma treatment notably depends on the type of

steel, a plasma source given by two opposite electrodes for plasma ignition and a pump periphery for evacuating the chamber. The plasma source is driven by a direct current (DC) power supply where the electrode distance, i.e. the discharge gap, is $120 \mathrm{~mm}$. For plasma treatment, the generator was operated at three different voltages and corresponding currents, resulting in three different powers dissipated in the plasma as listed in Tab. 1.

Tab. 1) Operating voltage and corresponding current including the resulting power dissipated in the plasma.

\begin{tabular}{|c|c|c|}
\hline Voltage $U$ in $\mathrm{V}$ & Current $/$ in $\mathrm{mA}$ & Power $P=U \cdot /$ in $\mathrm{W}$ \\
\hline 320 & 12 & 3.84 \\
\hline 420 & 24 & 10.08 \\
\hline 520 & 37 & 19.24 \\
\hline
\end{tabular}

the plasma source and discharge, respectively, and the corresponding particular operating factors. At fix plasma parameters, this approach features high efficiency and repeatability; moreover, the basic underlying effects and mechanisms are well understood. However, technical plasmas are wellknown to respond sensitively to slight changes in the operation parameters (e.g. operating voltage, pressure etc.) and the impact of variations in such parameters on the above-described glass modification was not yet investigated extensively. Against this background, the impact of the plasma power on the increase in absorption of plasma treated fused silica was investigated systematically in this work.

\section{Material and Methods}

In the present work, glass plates made of synthetic fused silica were examined. In order to achieve plasma-induced modification of the optical properties, the glass samples were treated in a homemade plasma generator. This plasma generator consists of a cylindrical vacuum chamber made of stainless
In the course of the plasma treatment, the pressure was kept constant at $p=70 \mathrm{~Pa}$ in all cases ${ }^{2}$. The plasma process gas was pure hydrogen $\left(\mathrm{H}_{2}\right)$ and treatment was performed in successive steps of 5 minutes each up to a total treatment duration of 30 minutes. In order to detect the treatment durationdependency of the plasma-induced increase in absorption, the transmission of the samples was measured after each step using an UV/VIS/NIR spectrometer (Lambda 1050 from PerkinElmer, Inc.).

Moreover, a further series of plasma treatment was performed where samples were exposed to the plasma for 30 minutes without any interruption. In the course of this treatment, the composition of the plasma within the vacuum chamber was monitored via emission spectroscopy employing an UV/VIS spectrometer (USB 2000 from Ocean Optics, Inc.). Here, spectra were taken at the beginning ( $t=0 \mathrm{~min}$.) and at the end of the plasma treatment (at $t=30 \mathrm{~min}$ ). Finally, the particular difference spectrum of both spectra was calculated in order to visualise and

\footnotetext{
1 Such plasma-induced modification of glass and the accompanying increase in near-surface absorption allows a reduction in laser energy required for ablation by a factor of 4.6 .

2 At this pressure, a medium vacuum is formed in the vacuum chamber. Since the mean free length of path of such a vacuum amounts to some millimetres, the number of collisions within the plasma volume and the accompanying de-excitation of plasma species are reduced.
} 
identify significant changes in plasma composition via comparing the line intensities of spectral lines of interest at the beginning and the end of the treatment process.

\section{Results and Discussion}

The plasma treatment procedure as described in Section 2 leads to notable increase in absorption as shown in Fig. 1. Here, the increase in absorption is visualised by the difference in transmission spectra of fused silica taken after and before plasma treatment for 30 minutes. plasma treatment. It further turns out that a significant increase in absorption is found in the ultraviolet wavelength range from approximately 200 to $400 \mathrm{~nm}$ for the lowest applied plasma power whereas this effect does not occur for higher plasma powers. This can be explained by a certain impact of the plasma power on the nature of the plasma as discussed below.

The drastic increase in absorption for the lowest plasma power shown in Fig. 1 can be attributed to a plasma-induced chemical reduction of silicon dioxide to substoichiometric oxygen-oxide com-

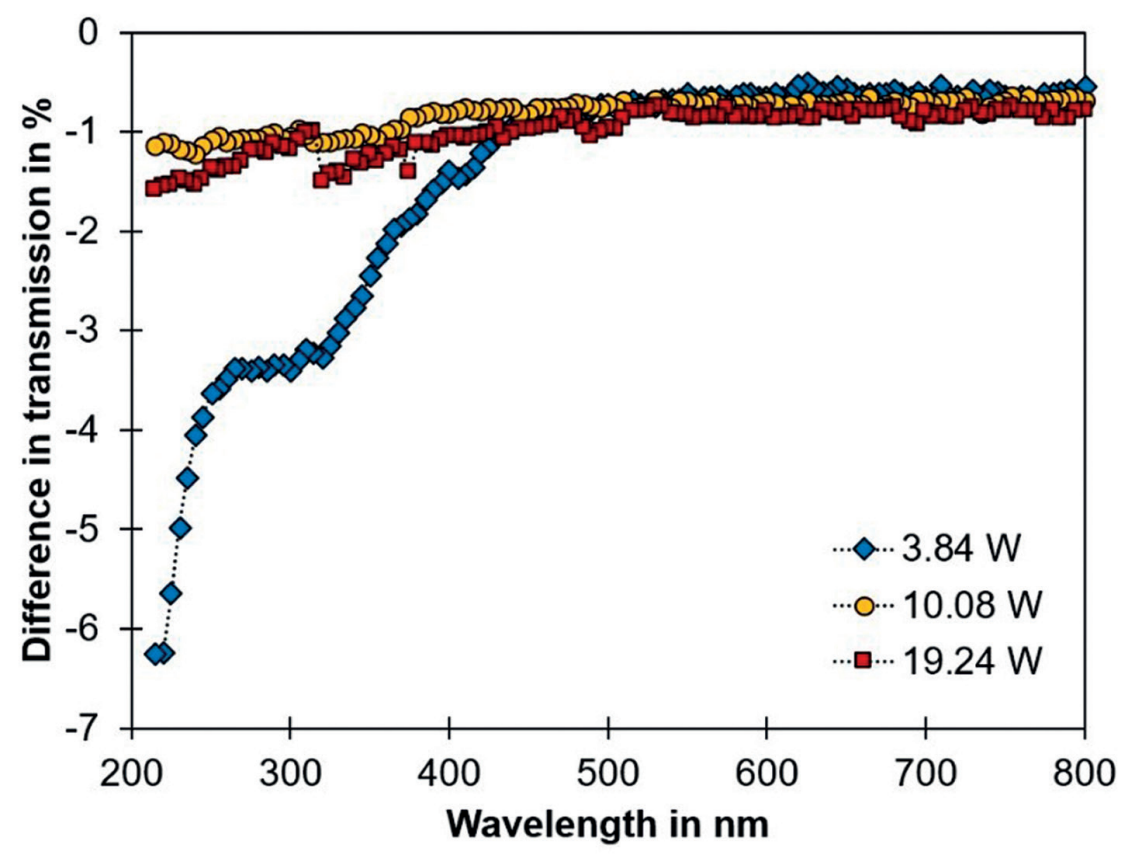

Fig. 1) Plasma-induced difference in transmission after plasma treatment for 30 minutes vs. wavelength for three different plasma powers.

Generally, an increase in absorption in the visible wavelength range by approximately $1 \%$ can be observed after

pounds and an additional implantation of hydrogen into the glass network as already confirmed via secondary ion

Tab. 2) Optically active defects in fused silica related to the deficiency of oxygen (data taken from (Skuja 1998)).

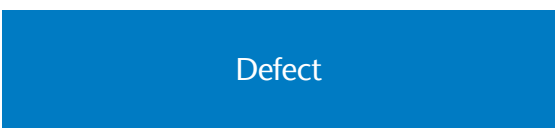

Oxygen deficiency centres

E'-centres

Hydrogen centres
Absorption peak positions in $\mathrm{nm}$

$229.60-243.11,393.60$

$196.80-206.64,213.77-217.52$
206.64-258.30 mass spectroscopy (SIMS) in previous work (Gerhard et al. 2012, Tasche et al. 2014). As listed in Tab. 2, these mechanisms lead to the formation of optically active defects such as oxygen deficiency centres, E'-centres or hydrogen centres which feature high absorption in the wavelength range concerned.

UV-absorbing oxygen deficiency centres are given by (i) simple and neutral oxygen vacancies or oxygen divacancies (Nishikawa et al. 1994), (ii) dicoordinated silicon (Skuja et al. 1984), and (iii) E'-centres (see Fig. 2), which are generally speaking unpaired electrons in silicon dioxide tetrahedrons ${ }^{3}$ due to oxygen vacancies, thus also referred to as positively charged oxygen vacancies (O'Reilly \& Robertson 1983). Finally, hydrogen centres or $\mathrm{H}(\mathrm{I})$-centres are formed by single hydrogen atoms attached to a silicon atom of a tetrahedron (Radtsig \& Bobyshev 1986), thus replacing an oxygen atom as shown in Fig. 2.

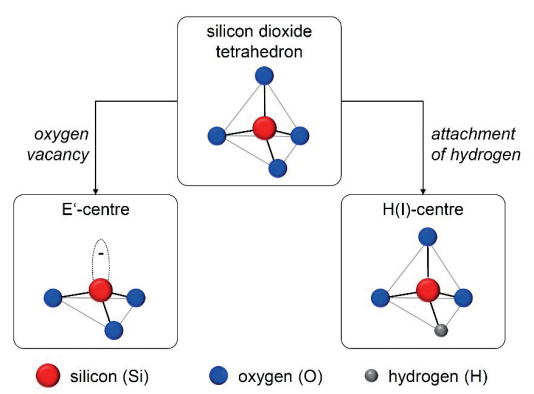

Fig. 2) Simplified visualisation of optically active $E^{\prime}$-centres and $H(I)$-centres within silicon dioxide tetrahedrons.

A chemical reduction in terms of a release of oxygen from the fused silica can easily be induced by hydrogenous plasma. By the plasma discharge, the process gas hydrogen $\left(\mathrm{H}_{2}\right)$ is dissociated by electron ( $\mathrm{e}^{-}$) impact according to

$$
H_{2}(g)+e^{-} \rightarrow 2 H(g)+e^{-}
$$

This process is quite easy to induce at low power since the hydrogen molecule features a low dissociation energy of merely $4.5 \mathrm{eV}$. Plasma-induced dissociation thus leads to the formation of atomic hydrogen $(\mathrm{H})$ which features an extremely high chemical reactivity (Shirai et al. 2002). When getting in contact

3 The basic structural element of silicon dioxide and fused silica is a tetrahedron consisting of one silicon atom and four oxygen atoms where each oxygen atom appertains to two silicon atoms. 
with the fused silica $\left(\mathrm{SiO}_{2}\right)$ surface, a possible reaction is the formation of silicon monoxide $(\mathrm{SiO})$ and water $\left(\mathrm{H}_{2} \mathrm{O}\right)$, following from

$$
\mathrm{SiO}_{2}(s)+2 \mathrm{H}(\mathrm{g}) \rightarrow \mathrm{SiO}(s)+\mathrm{H}_{2} \mathrm{O}(\mathrm{g})(2) .
$$

Consequently, oxygen is removed from the glass matrix and the absorption in the ultraviolet wavelength range is increased. In the present case, such chemical reduction was confirmed by emission spectroscopic diagnostic of the plasma during the treatment process. For this purpose, difference spectra were calculated as described in Section 2. As shown in Fig. 3, a notable increase in the line intensity of two characteristic oxygen species, i.e. singly ionised oxygen (OII) at an emission wavelength of $376.25 \mathrm{~nm}$ (Wenåker 1990) and doubly ionised oxygen (OIII) at an emission wavelength of $745.54 \mathrm{~nm}$ (Luo et al. 1989), can be observed in the course of the plasma treatment process.

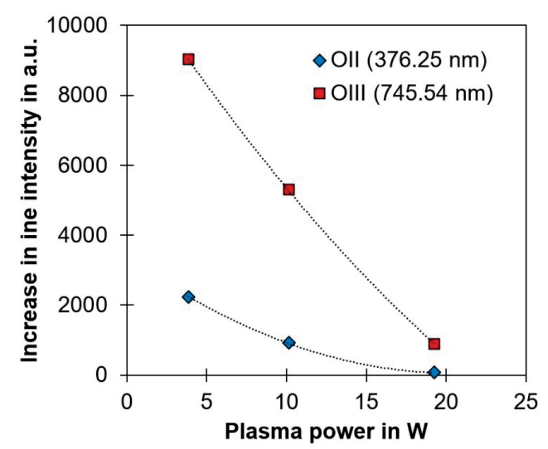

Fig. 3) Increase in line intensity of two selected oxygen species, OII and OIII, given by the difference of the particular line intensities at the end and the beginning of the treatment process vs. plasma power.

It can be stated that in any case, an increase in oxygen concentration in the plasma volume within the vacuum chamber was detected where the oxygen most likely originates from the glass network forming silicon dioxide. It can further be seen that the oxygen concentration increases with decreasing plasma power. This behaviour correlates well with the plasma-induced decrease in transmission where the highest increase is found for the lowest plasma power as shown in Fig. 4a).

Here, the dependency of the glass transmission on the plasma treatment duration for the three applied plasma powers is shown at a discrete wavelength of $248 \mathrm{~nm}$. This wavelength was chosen since the corresponding laser source, the krypton fluoride (KrF) excimer laser, is well-established and commonly used in lasers materials processing.

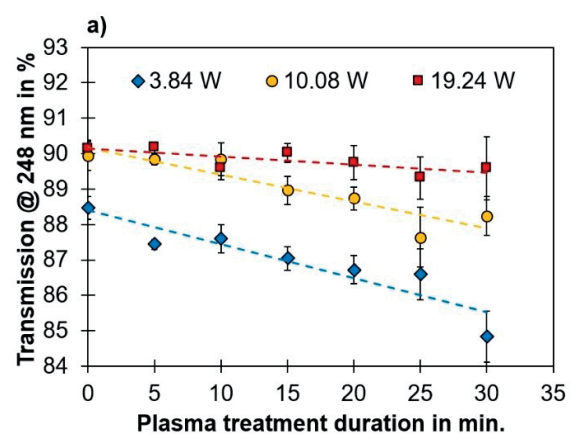

Fig. 4) Transmission at $248 \mathrm{~nm}$ vs. plasma treatment duration for three different plasma powers (a) and slope of the particular linear regression lines vs. plasma power (b).

When taking a closer look on the trend as indicated by the particular linear regression lines ${ }^{4}$, it turns out that the slope of these lines directly represents the differences in plasma treatment efficiency where the highest steepness is found for the lowest plasma power, see Fig. 4b). This obvious decrease in plasma treatment efficiency with increasing plasma power is an interesting point and should be taken into account in practice. It confirms the well-known fact that for plasma-based processes the ideal working range is strongly power-dependent and that the application of high plasma powers or energies may even lead to contrary and unwanted effects and results.

From a physical point of view, the behaviour observed in the present case can be explained by a reduction of the plasma species needed for inducing optically active defects with increasing plasma power due to two different and interacting main effects: First, the by-product of the underlying chemical reaction is water as expressed by Eq. 2. This molecule is based on a covalent binding, a.k.a. atomic bond, where electrons are shared by the

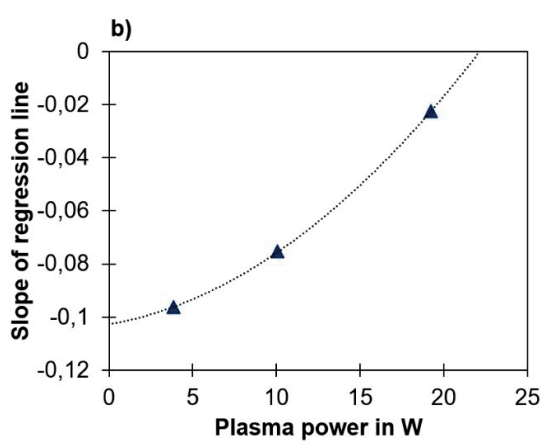

involved atoms, oxygen and water. When increasing the plasma power, the ionisation degree of the plasma is increased as well ${ }^{5}$. Thus, the amount of atomic hydrogen is decreased whereas the amount of ionised hydrogen is increased. Consequently, the concentration of the actually needed species for hydrogen-induced chemical reduction, the electrically neutral hydrogen and the treatment efficiency decreases. Second, increasing the discharge voltage and plasma power, respectively, leads to an increase in the electric field strength $E$ within the plasma volume. This may lead to an increase in losses of electrons at walls due to drift and diffusion as described by the drift-diffusion-approximation (Becker \& Loffhagen 2013), given by

$$
J_{e}=-n_{e} \cdot \mu_{e} \cdot E-D \cdot \Delta c_{e}
$$

According to this model, the electron flux $J_{e}$ depends on the number of electrons $n_{e}$, the electron mobility $\mu_{e}$, the electric field strength $E$, the diffusion coefficient $D$ and the electron concentration gradient $\Delta c_{e}$. Here, the expression $n_{e} \cdot \mu_{e} \cdot E$ denotes the drift component and the second term, $D \cdot \Delta c$, represents diffusion. The mechanisms of drift and diffusion finally lead to ambipolar diffusion of negatively and positively charged plasma species, i.e. electrons and ions, towards the wall of the vacuum chamber of the plasma generator. In case of hydrogen, this process is approximately 43-times faster for electrons than for ions ${ }^{6}$. Thus, electrons are

4 The approach of linear regression was chosen due to the fact that a hydrogen plasma-induced change in transmission and the plasma treatment duration feature a linear interrelationship (Gerhard et al. 2013)

5 For comparison, the ionisation energy $(13.6 \mathrm{eV})$ of hydrogen is approximately 3 times higher than its dissociation energy $(4.5 \mathrm{eV})$.

6 The minimum value of the ion-electron velocity ratio is given by the square root of the ratio of the ion mass $\left(1.67 \times 10^{-27} \mathrm{~kg}\right)$ and the electron mass $\left(9,11 \times 10^{-31} \mathrm{~kg}\right)$. 
preferentially removed from the plasma volume (Phelps 1990) and cannot contribute to the dissociation of hydrogen molecules via electron impact. An effect, which further contributes to the observed decrease in efficiency by reducing the number of hydrogen atoms within the plasma when increasing the plasma power.

\section{Conclusions}

As shown by the results of the present work, the electrical power dissipated in a plasma has a significant impact on the efficiency of plasma treatment for increasing the absorption of fused silica. It was shown that absorption can be increased efficiently when applying plasma treatment at comparatively low power whereas the application of higher powers leads to poor results in terms of decrease in transmission. This effect is most likely due to a reduction in concentration of plasma species required for initiating the wanted effect, i.e. the formation of optically active glass defects such as oxygen vacancies as well as the formation of $\mathrm{E}^{\prime}$ - and $\mathrm{H}(\mathrm{I})$-centres by atomic hydrogen provided by the plasma. The resulting plasma-induced increase in absorption allows improving the quality and efficiency of laserbased glass structuring and machining methods and is thus an advantageous effect in this context. Even though this approach was already investigated and reported in previous work the present contribution is the first study on the impact of the plasma power on the plasma treatment process. It is thus a valuable and important basis for further detailed investigations of the underlying mechanisms and effects which occur during plasma-induced modification of optical properties of glasses.

\section{REFERENCES}

Becker MM, Loffhagen D (2013) Enhanced reliability of drift-diffusion approximation for electrons in fluid models for nonthermal plasmas. AIP Advances 3(1):12108. doi: $10.1063 / 1.4775771$

Böhme R, Zimmer K (2004) Low roughness laser etching of fused silica using an adsorbed layer. Appl Surf Sci 239(1):109-116. doi: 10.1016/j.apsusc.2004.05.095

Brückner S, Hoffmeister J, Ihlemann J, Gerhard C, Wieneke S, Viöl W (2012) Hybrid Laser-Plasma Micro-Structuring of Fused Silica Based on Surface Reduction by a Low-Temperature Atmospheric Pressure Plasma. JLMN 7(1):73-76. doi: 10.2961/jlmn.2012.01.0014

Gerhard C, Dammann M, Wieneke S, Viöl W (2014) Sequential Atmospheric Pressure Plasma-Assisted Laser Ablation of Photovoltaic Cover Glass for Improved Contour Accuracy. Micromachines 5(3):408-419.

doi: 10.3390/mi5030408

Gerhard C, Tasche D, Brückner S, Wieneke S, Viöl W (2012) Near-surface modification of optical properties of fused silica by low-temperature hydrogenous atmospheric pressure plasma. Opt Lett 37(4):566-568. doi: 10.1364/OL.37.000566

Gerhard C, Weihs T, Tasche D, Brückner S, Wieneke S, Viöl W (2013) Atmospheric Pressure Plasma Treatment of Fused Silica, Related Surface and Near-Surface Effects and Applications. Plasma Chem Plasma Process 33(5):895905. doi: 10.1007/s11090-013-9471-7

Hoffmeister J, Gerhard C, Brückner S, Ihlemann J, Wieneke S, Viöl W (2012) Laser Micro-Structuring of Fused Silica Subsequent to Plasma-Induced Silicon Suboxide Generation and Hydrogen Implantation. Physics Procedia 39:613-620. doi: 10.1016/j.phpro.2012.10.080

Hopp B, Vass C, Smausz T, Bor Z (2006) Production of submicrometre fused silica gratings using laser-induced backside dry etching technique. I. Phys. D: Appl. Phys. 39(22):4843-4847. doi: 10.1088/0022-3727/39/22/015

Luo D, Pradhan AK, Saraph HE, Storey PJ, Yu Y (1989) Atomic data for opacity calculations. X. Oscillator strengths and photoionisation cross sections for O III. J. Phys. B: At. Mol. Opt. Phys. 22(3):389-406 doi: $10.1088 / 0953-4075 / 22 / 3 / 006$

Nishikawa H, Watanabe E, Ito D, Ohki Y (1994) Decay kinetics of the 4.4-eV photoluminescence associated with the two states of oxygen-deficient-type defect in amorphous SiO2. Phys Rev Lett 72(13):2101-2104. doi: 10.1103/PhysRevLett.72.2101

O'Reilly EP, Robertson J (1983) Theory of defects in vitreous silicon dioxide. Phys. Rev. B 27(6):3780-3795. doi: 10.1103/PhysRevB.27.3780

Phelps AV (1990) The Diffusion of Charged Particles in Collisional Plasmas: Free and Ambipolar Diffusion at Low and Moderate Pressures. J Res Natl Inst Stand Technol 95(4):407-431. doi: 10.6028/jres.095.035

Radtsig VA, Bobyshev AA (1986) Twofold coordinated $\mathrm{Si}$ and Ge atoms and "hydrogen" paramagnetic centers in amorphous $\mathrm{SiO} 2$ and $\mathrm{GeO} 2$. phys. stat. sol. (b) 133(2):621-627. doi: 10.1002/pssb.2221330223

Shirai H, Fujimura Y, Jung S (2002) Formation of nanocrystalline silicon dots from chlorinated materials by RF plasma-enhanced chemical vapor deposition. Thin Solid Films 407(1-2):12-17. doi: 10.1016/50040-6090(02)00005-6

Skuja L (1998) Optically active oxygen-deficiency-related centers in amorphous silicon dioxide.

J Non-Crystalline Solids 239(1-3):16-48.

doi: 10.1016/S0022-3093(98)00720-0

Skuja LN, Streletsky AN, Pakovich AB (1984) A new intrinsic defect in amorphous SiO2: Twofold coordinated silicon. Solid State Communications 50(12):1069-1072. doi: 10.1016/0038-1098(84)90290-4
Tasche D, Gerhard C, Ihlemann J, Wieneke S, Viöl W (2014) The impact of O/Si ratio and hydrogen content on ArF excimer laser ablation of fused silica. JEOS:RP 9. doi: 10.2971/jeos.2014.14026

Utéza O, Sanner N, Chimier B, Brocas A, Varkentina N, Sentis M, Lassonde $P$, Légaré $F$, Kieffer IC (2011) Control of material removal of fused silica with single pulses of few optical cycles to sub-picosecond duration. Appl Phys A Mater Sci Process 105(1):131-141. doi: 10.1007/s00339. 011-6469-y

Wang J, Niino H, Yabe A (1999) One-step microfabrication of fused silica by laser ablation of an organic solution. Appl Phys A Mater Sci Process 68(1):111-113. doi: $10.1007 / \mathrm{s} 003390050863$

Wenåker I (1990) The spectrum of singly ionized oxygen, O II. Phys. Scr. 42(6):667-684. doi: 10.1088/0031$8949 / 42 / 6 / 008$

\section{AUTHORS}

Prof. Dr. rer. nat. Christoph Gerhard

Dr. rer. nat. Mandy Hofmann

Fachbereich Ingenieur- und Naturwissenschaften

Technische Hochschule Wildau

Emilie Letien

Institut Universitaire de Technologie A de Lille

Département Mesures Physiques

Thomas Cressent

Institut Universitaire de Technologie A de Lille

Département Chimie

E-Mail for correspondence :

christoph.gerhard@th-wildau.de

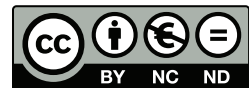

